\title{
ROTATIONAL-VIBRATIONAL EIGEN SOLUTIONS OF THE D-DIMENSIONAL SCHRÖDINGER EQUATION FOR THE IMPROVED WEI POTENTIAL
}

\author{
${ }^{* 1}$ Eyube, E. S., ${ }^{2}$ Yabwa, D. and ${ }^{3}$ Wadata, $U$. \\ ${ }^{1}$ Department of Physics, School of Physical Sciences, Modibbo Adama University of Technology, Yola, Nigeria \\ ${ }^{2}$ Department of Physics, Faculty of Science, Taraba State University, Jalingo, Nigeria \\ ${ }^{3}$ Department of Physics, School of Sciences, Aminu Saleh College of Education, Azare, Nigeria \\ *Corresponding Author: Email Address: edwineyubes@ mautech.edu.ng; Tel.: +2348036870057
}

\begin{abstract}
In this present study, we have employed the techniques of exact quantization rule and ansatz solution method to obtain closed form expressions for the rotational-vibrational eigensolutions of the Ddimensional Schrödinger equation for the improved Wei potential, for cases of $h^{\prime} \neq 0$ and $h^{\prime}=0$. By using our derived energy equation and choosing arbitrary values of $\mathrm{n}$ and $\ell$, we have computed the bound state rotational-vibrational energies of $\mathrm{CO}, \mathrm{H}_{2}$ and $\mathrm{LiH}$ for various quantum states. The mean absolute percentage deviation (MAPD) and the Lippincott criterion ware used as a goodness-of-fit indices to compare our result with the Rydberg-Klein-Rees (RKR) and improved Tietz potential data in the literature. MAPD of $0.2862 \%, 0.2896 \%$ and $0.0662 \%$ relative to the RKR data for CO ware obtained. For the improved Wei and Morse potential, our computed energy eigenvalues for $\mathrm{CO}, \mathrm{H}_{2}$ and $\mathrm{LiH}$ are in excellent agreement with existing results in the literature.
\end{abstract}

Keywords: Improved Wei potential, Morse potential, exact quantization rule, ansatz solution, RKR data, D-dimensions

\section{INTRODUCTION}

Extensive literature review reveals that wave functions are of tremendous importance in both relativistic and nonrelativistic quantum mechanics because they completely define the quantum mechanical system under review (Yanar et al., 2020; Hamzavi et al., 2012), information such as energy of the system, momentum, frequency of vibration, speed and wavelength are readily obtainable if the wave function of the system is known (Eyube et al., 2019a). Obtaining the wave function of a quantum mechanical system requires solving the Schrödinger equation for a given potential energy function (Eyube et al., 2019b). The Coulomb and harmonic oscillator potentials are known to give exact solution with the Schrödinger equation for all quantum states $\mathrm{n} \ell$ (Qiang et al., 2009) where $n$ is the vibrational quantum number and $\ell$ is the rotational quantum number, similarly, few other potential energy functions such as Eckart, Hulthén and Morse potentials give exact solution only for the s-wave $(\ell=0)$ state (Ikhdair, 2011; Serrano et al., 2010). Most of the other known potential energy models have no exact solution with the Schrödinger equation for all values of $\mathrm{n}$ and $\ell$, with these class of potential energy functions only approximate numerical (Nasser et al., 2012; Lucha and Schöberl, 1999) or approximate analytical (Khodja et al., 2019; Eyube et al., 2019c; Ferreira and Bezerra, 2017) solutions are possible. Solving the Schrödinger equation by approximate analytical method is quite a challenge, it involves applying an appropriate approximation scheme (Ferreira and Prudente, 2017; Greene and Aldrich, 1976; Pekeris, 1934) to deal with the centrifugal term of the effective potential energy function, followed by a suitable solution technique. Different solution methods have been used by researchers to solve the Schrödinger equation, some of the solution techniques include: asymptotic iteration method (Falaye et al., 2013), generalized pseudospectral method (Roy, 2013), exact

$$
\psi_{n \ell}^{\prime \prime}(x)+k_{n \ell}^{2}(x) \psi_{n \ell}(x)=0
$$

quantization rule (Falaye et al., 2015; Ikhdair and Sever, 2009; $\mathrm{Ma}$ and $\mathrm{Xu}, 2005$ ), proper quantization rule (Louis et al., 2019; Dong and Cruz-Irrison, 2012) path integral approach (Khodja et al., 2019), Laplace transform approach (Tsaur and Wang, 2014), Nikiforov-Uvarov method (Khordad and Mirhosseini, 2015; Ikot et al., 2013; Yazarloo et al., 2012) and ansatz solution method (Okorie et al., 2020; Tang et al., 2013). In the year 1990, Wei proposed a four parameter potential energy function which fits the experimental Rydberg-Klein-Rees (RKS) data more closely than the Morse potential, particularly when the potential domain extends to near the dissociation limit (Jia et al., 2012), the Wei potential has been used to investigate the rotationalvibrating levels of diatomic molecules (Kunc and GordilloVazquez, 1997). However, it is pertinent to note that the expressions derived by these authors for the energy eigenvalues and eigenfunctions are only restricted to the case $h^{\prime} \neq 0$ as these expressions become infinite when $h^{\prime}=0$ which is not physically acceptable for a finite potential energy function. In the present study, we aimed at obtaining closed form expressions for the rotational-vibrational eigensolutions of the D-dimensional Schrödinger equation for the improved Wei potential via exact quantization rule (EQR) and ansatz solution method. As our specific objectives, we will consider cases of rotational-vibrational energies and radial wave functions for $\mathrm{h}^{\prime} \neq 0$ as well as $\mathrm{h}^{\prime}=0$, we will compare results with those in the literature where they exist.

\section{THEORETICAL APPROACH}

\section{Overview of the concepts of exact quantization rule}

Here we present in outline form, the basic concepts of EQR, a detailed description of the concept is given by Ma and $\mathrm{Xu}$ (2005). The EQR has been proposed to solve the onedimensional Schrödinger equation given by:

where 


$$
k_{n \ell}(x)=\sqrt{\frac{2 \mu}{\hbar^{2}}\left\{E_{n \ell}-V_{e f f}(x)\right\}}
$$

is the momentum of the system, $\mu$ as the mass, $E_{n \ell}$ is the energy eigenvalue, $V_{e f f}(x)$ is the effective potential energy function which is a piecewise continuous real function of $x$ and $\psi_{n \ell}(x)$ is the wave function. If we define the phase angle, $\varphi_{n \ell}(x)$ as:

$$
\varphi_{n \ell}(x)=\psi_{n \ell}^{\prime}(x) / \psi_{n \ell}(x)
$$

Eq. (1) assumes the well-known Riccati differential equation given by:

$$
\varphi_{n \ell}^{\prime}(x)+\frac{2 \mu}{\hbar^{2}}\left\{E_{n \ell}-V_{e f f}(x)\right\}+\varphi_{n \ell}^{2}(x)=0
$$

As a result of Sturm-Liouville theorem, $\varphi_{n \ell}(x)$ decreases monotonically with respect to $x$ between two turning points determined by the equation $E_{n \ell} \geq V_{e f f}(x)$. Particularly, $x$ increases across a node of the wavefunction $\psi_{n \ell}(x)$, where $E_{n \ell} \geq V_{e f f}(x), \varphi_{n \ell}(x)$ decreases to $-\infty$ and jumps to $+\infty$ and then decreases again. By carefully studying the onedimensional Schrödinger equation, Ma and Xu (2005) proposed an EQR given by:

$$
\int_{x_{n A}}^{x_{n B}} k_{n \ell}(x) d x=N \pi+\int_{x_{n A}}^{x_{n B}} \varphi_{n \ell}(x)\left[\frac{d k_{n \ell}(x)}{d x}\right]\left[\frac{d \varphi_{n \ell}(x)}{d x}\right]^{-1} d x
$$

where $x_{n A}$ and $x_{n B}$ are two turning points determined by solving the equation $E_{n \ell}=V_{e f f}(x)$ and $x_{n A}<x_{n B} . \mathrm{N}$ is the number of nodes of $\varphi_{n \ell}(x)$ in the neighborhood of $\mathrm{E}_{\mathrm{n} \ell} \geq \mathrm{V}_{\text {eff }}(\mathrm{x})$ and it is larger by one than the number of nodes $\mathrm{n}$ of the wavefunction $\psi_{n \ell}(x)$, clearly, $\mathrm{N}=\mathrm{n}+1$. The first term, $\mathrm{N} \pi$, is the contribution from the nodes of the wave function, and the second term is referred to as the quantum correction. The quantum correction is independent of the number of nodes for the exactly solvable systems ( $\mathrm{Ma}$ and $\mathrm{Xu}, 2005)$, therefore, it can be evaluated for the ground state $(\mathrm{n}=0)$, the second term in Eq. (5) can thus be represented by:

$$
Q_{c}=\int_{x_{n A}}^{x_{n B}} \varphi_{n \ell}(x)\left[\frac{d k_{n \ell}(x)}{d x}\right]\left[\frac{d \varphi_{n \ell}(x)}{d x}\right]^{-1} d x \equiv \int_{x_{0 A}}^{x_{0 B}} \varphi_{0 \ell}(x)\left[\frac{d k_{0 \ell}(x)}{d x}\right]\left[\frac{d \varphi_{0 \ell}(x)}{d x}\right]^{-1} d x
$$

where $Q_{c}$ is the quantum correction term. In three dimensional spherical coordinates, the EQR is given by:

$$
\int_{r_{n A}}^{r_{n B}} k_{n \ell}(r) d r=N \pi+\int_{r_{n A}}^{r_{n B}} \varphi_{0 \ell}(r)\left[\frac{d k_{0 \ell}(r)}{d r}\right]\left[\frac{d \varphi_{0 \ell}(r)}{d r}\right]^{-1} d r
$$

In simplified form, Eq. (6) can be expressed as:

$$
I=N \pi+Q_{c}
$$

where the momentum integral is given by:

$$
I=\int_{r_{n A}}^{r_{n B}} k_{n \ell}(r) d r
$$

and the quantum correction is:

$$
Q_{c}=\int_{r_{n A}}^{r_{n} B} \phi_{0 \ell}(r)\left[\frac{d k_{0 \ell}(r)}{d r}\right]\left[\frac{d \phi_{0 \ell}(r)}{d r}\right]^{-1} d r
$$

The radial Schrödinger equation in D-dimensional space Falaye et al. (2015) is written as:

$$
\frac{d^{2} \psi_{n \ell}(r)}{d r^{2}}+\frac{2 \mu}{\hbar^{2}}\left\{E_{n \ell}-V_{e f f}(r)\right\} \psi_{n \ell}(r)=0
$$

$\psi_{n \ell}(r)$ is the radial wave function, $r$ is the internuclear separation and $V_{e f f}(r)$ is the effective potential defined in terms of a spherically symmetric potential $V(r)$ and a parameter $\Lambda$ by: 


$$
V_{e f f}(r)=V(r)+\frac{\left(\Lambda^{2}-1 / 4\right) \hbar^{2}}{2 \mu r^{2}}
$$

with $\Lambda$ given in terms of spatial dimensions, $\mathrm{D} \geq 2$ as:

$$
\Lambda=\ell+\frac{1}{2}(D-2)
$$

\section{Rotational-vibrational energy of the improved Wei potential}

By choosing $V(r)$ to be the improved Wei potential (Jia et al., 2012):

$$
V(r)=D_{e}\left(1-\frac{a}{e^{b r}-H}\right)^{2}
$$

where $D_{e}$ is the dissociation energy, $r_{e}$ is the molecular bond length, $r$ is the internuclear separation, $H=h^{\prime} e^{b r_{e}}$ and $a=\left(1-h^{\prime}\right) e^{b r_{e}}$. The parameters $h^{\prime}$ (dimensionless) and $b \quad$ (in $\mathrm{m}^{-1}$ ) are determined through $f_{2}$ and $f_{3}$ the second and third derivatives of the potential energy function (Eq. (14)) at $r=r_{e}$ respectively (Jia et al., 2012), therefore, by using the following relationship

$$
\frac{d^{2} V(r)}{d r^{2}}=f_{2}=4 \pi^{2} \mu c^{2} \omega_{e}^{2}
$$

where $\omega_{e}$ is the equilibrium vibrational harmonic frequency $\left(\right.$ in $\mathrm{m}^{-1}$ ), we find:

$$
h^{\prime}=1-\frac{b}{\pi c \omega_{e}}\left(\frac{D_{e}}{2 \mu}\right)^{\frac{1}{2}}
$$

also, employing the relationship between the vibrational-rotational coupling constant, $\alpha_{e}$ and $f_{3}$ (Jia et al., 2012), viz:

$$
\alpha_{e}=-\frac{6 B_{e}^{2}}{\omega_{e}}\left(1+\frac{r_{e} f_{3}}{3 f_{2}}\right)
$$

$B_{e}$ being the rotational constant given by (Jia et al., 2012):

$$
B_{e}=\frac{\hbar}{4 \pi c \mu r_{e}^{2}}
$$

using Eqs. (16) - (18) and obtaining $f_{3}$ from (14), get:

$$
b=\pi c \omega_{e}\left(\frac{8 \mu}{D_{e}}\right)^{\frac{1}{2}}-\frac{8 \pi^{2} c^{2} \mu^{2} r_{e}^{3} \alpha_{e} \omega_{e}}{3 \hbar^{2}}-\frac{1}{r_{e}}
$$

Putting Eq. (14) in (12), we have the effective improved Wei potential given by:

$$
V_{e f f}(r)=D_{e}\left(1-\frac{a}{e^{b r}-H}\right)^{2}+\frac{\left(\Lambda^{2}-1 / 4\right) \hbar^{2}}{2 \mu r^{2}}
$$

Eq. (1) when used with the effective potential of Eq. (20) has exact solution only for the s-wave $(\ell=0)$ case, to obtain analytical solution for all quantum states, we must use a suitable approximation scheme to deal with the centrifugal or spin-orbit term, $1 / r^{2}$ is approximated by a Pekeris-type approximation (Hamzavi et al., 2012) given as:

$$
\frac{1}{r^{2}} \approx \frac{1}{r_{e}^{2}}\left\{c_{0}+\frac{c_{1}}{e^{b r}-H}+\frac{c_{2}}{\left(e^{b r}-H\right)^{2}}\right\}
$$

where $c_{0}, c_{1}$ and $c_{2}$ are adjustable parameters given by:

$$
\begin{aligned}
& c_{0}=1+\frac{1}{b r_{e}}\left(1-h^{\prime}\right)\left(3+h^{\prime}\right)-\frac{3}{b^{2} r_{e}^{2}}\left(1-h^{\prime}\right)^{2} \\
& c_{1}=\frac{2 e^{b r_{e}}}{b r_{e}}\left(1-h^{\prime}\right)^{2}\left(2+h^{\prime}\right)-\frac{6 e^{b r_{e}}}{b^{2} r_{e}^{2}}\left(1-h^{\prime}\right)^{3}
\end{aligned}
$$




$$
c_{2}=-\frac{e^{2 b r_{e}}}{b r_{e}}\left(1-h^{\prime}\right)^{3}\left(1+h^{\prime}\right)+\frac{3 e^{2 b r_{e}}}{b^{2} r_{e}^{2}}\left(1-h^{\prime}\right)^{4}
$$

Substituting Eq. (21) in Eq. (20), this leads to:

$$
V_{e f f}(r)=D_{e}\left(1-\frac{a}{e^{b r}-H}\right)^{2}+\frac{\left(\Lambda^{2}-1 / 4\right) \hbar^{2}}{2 \mu r_{e}^{2}}\left\{c_{0}+\frac{c_{1}}{e^{b r}-H}+\frac{c_{2}}{\left(e^{b r}-H\right)^{2}}\right\}
$$

the substitution:

$$
x=\left(e^{b r}-H\right)^{-1} .
$$

transforms Eq. (25) transforms to:

$$
V_{e f f}(x)=D_{e}(1-a x)^{2}+\frac{\left(\Lambda^{2}-\frac{1}{4}\right) \hbar^{2}}{2 \mu r_{e}^{2}}\left(c_{0}+c_{1} x+c_{2} x^{2}\right)
$$

Eq. (27) can be expressed in polynomial form as:

$$
\begin{aligned}
V_{e f f}(x)=\frac{\hbar^{2}}{2 \mu r_{e}^{2}}\left\{\left(\Lambda^{2}-\frac{1}{4}\right) c_{2}+\frac{2 \mu r_{e}^{2} D_{e} a^{2}}{\hbar^{2}}\right\} x^{2}+ & \frac{\hbar^{2}}{2 \mu r_{e}^{2}}\left\{\left(\Lambda^{2}-\frac{1}{4}\right) c_{1}-\frac{4 \mu r_{e}^{2} D_{e} a}{\hbar^{2}}\right\} x \\
& +\frac{\hbar^{2}}{2 \mu r_{e}^{2}}\left\{\left(\Lambda^{2}-\frac{1}{4}\right) c_{0}+\frac{2 \mu r_{e}^{2} D_{e}}{\hbar^{2}}\right\}
\end{aligned}
$$

by defining the following parameters:

$$
\begin{aligned}
& \varepsilon_{1}^{2}=\left(\Lambda^{2}-\frac{1}{4}\right) c_{2}+\frac{2 \mu r_{e}^{2} D_{e} a^{2}}{\hbar^{2}} \\
& \varepsilon_{2}^{2}=\left(\Lambda^{2}-\frac{1}{4}\right) c_{1}-\frac{4 \mu r_{e}^{2} D_{e} a}{\hbar^{2}} \\
& \varepsilon_{3}^{2}=\left(\Lambda^{2}-\frac{1}{4}\right) c_{0}+\frac{2 \mu r_{e}^{2} D_{e}}{\hbar^{2}} \\
& \alpha=\frac{\hbar^{2} \varepsilon_{1}^{2}}{2 \mu r_{e}^{2}} \\
& \beta=\frac{\hbar^{2} \varepsilon_{2}^{2}}{2 \mu r_{e}^{2}} \\
& \gamma=\frac{\hbar^{2} \varepsilon_{3}^{2}}{2 \mu r_{e}^{2}}
\end{aligned}
$$

in compact form, Eq. (28) can be expressed in the form:

$$
V_{e f f}(x)=\alpha x^{2}+\beta x+\gamma
$$

To enable determine the Riccati equation (Gu and Dong, 2011), first, we need to obtain two turning points $x_{n A}$ and $x_{n B}$ by solving the equation:

$$
V_{e f f}(x)=E_{n \ell}
$$

Inserting Eq. (35) in Eq. (36), we have that:

$$
\alpha x^{2}+\beta x+\gamma-E_{n \ell}=0
$$

$x_{n A}$ and $x_{n B}\left(>x_{n A}\right)$ being the roots Eq. (37) are given by:

$$
x_{n A}=-\frac{\beta+\sqrt{\beta^{2}-4 \alpha\left(\gamma-E_{n \ell}\right)}}{2 \alpha}
$$

and 


$$
x_{n B}=-\frac{\beta-\sqrt{\beta^{2}-4 \alpha\left(\gamma-E_{n \ell}\right)}}{2 \alpha}
$$

using Eqs. (38) and (39), the sum and products of $x_{n A}$ and $x_{n B}$ which may be required later are given respectively by:

$$
\begin{aligned}
& x_{n A}+x_{n B}=-\frac{\beta}{\alpha} . \\
& x_{n A} x_{n B}=\frac{\gamma-E_{n \ell}}{\alpha} .
\end{aligned}
$$

for the ground ( $n=0$ ) state, Eq. (40) and (41) gives respectively:

$$
\begin{aligned}
& x_{0 A}+x_{0 B}=-\frac{\beta}{\alpha} . \\
& x_{0 A} x_{0 B}=\frac{\gamma-E_{0 \ell}}{\alpha} .
\end{aligned}
$$

substituting Eq. (35) in the momentum relation given by Eq. (2), this gives:

$$
k_{n \ell}(x)=\sqrt{\frac{2 \mu}{\hbar^{2}}\left(E_{n \ell}-\alpha x^{2}-\beta x-\gamma\right)} .
$$

an important result for the momentum is one which involves the turning points, thus, if we write Eq. (44) as in the following form:

$$
k_{n \ell}(x)=\sqrt{\frac{2 \mu \alpha}{\hbar^{2}}\left(\frac{E_{n \ell}-\gamma}{\alpha}-x^{2}-\frac{\beta}{\alpha} x\right)} .
$$

inserting Eq. (32), (40) and (41), this gives:

$$
k_{n \ell}(x)=\frac{\varepsilon_{1}}{r_{e}} \sqrt{\left(x-x_{n A}\right)\left(x_{n B}-x\right)} .
$$

differentiating Eq. (46) with respect to $x$ as this will be required in evaluating the quantum correction for the ground state, this gives:

$$
k_{n \ell}^{\prime}(x)=-\frac{\varepsilon_{1}}{r_{e}} \frac{x+\beta / 2 \alpha}{\sqrt{\left(x-x_{n A}\right)\left(x_{n B}-x\right)}} .
$$

The Riccati equation given by Eq. (4), in three dimensional spherical coordinates is written as (Ikhdair and Sever, 2009):

$$
\varphi_{n \ell}^{\prime}(r)+\frac{2 \mu}{\hbar^{2}}\left\{E_{n \ell}-V_{e f f}(r)\right\}+\varphi_{n \ell}^{2}(r)=0 .
$$

using Eq. (26) followed by Eq. (35) in Eq. (48), the Riccati equation in terms of variable $x$ is therefore given by:

$$
-b x(1+H x) \varphi_{n \ell}^{\prime}(x)+\frac{2 \mu}{\hbar^{2}}\left(E_{n \ell}-\alpha x^{2}-\beta x-\gamma\right)+\varphi_{n \ell}^{2}(x)=0
$$

which for the ground state $(n=0)$ gives;

$$
-b x(1+H x) \varphi_{0 \ell}^{\prime}(x)+\frac{2 \mu}{\hbar^{2}}\left(E_{0 \ell}-\alpha x^{2}-\beta x-\gamma\right)+\varphi_{0 \ell}^{2}(x)=0
$$

since $\varphi_{0 \ell}(x)$ has one zero and no pole, we choose a linear polynomial as a trial solution, assume:

$$
\varphi_{0 \ell}(x)=-m x+c .
$$

where $\mathrm{m}$ and $\mathrm{c}$ are constants, substituting Eq. (51) in Eq. (50), get:

$$
m b\left(x+H x^{2}\right)+\frac{2 \mu}{\hbar^{2}}\left(E_{0 \ell}-\alpha x^{2}-\beta x-\gamma\right)+m^{2} x^{2}-2 c m x+c^{2}=0
$$

which simplifies to;

$$
\left(m b H-\frac{2 \mu \alpha}{\hbar^{2}}+m^{2}\right) x^{2}+\left(m b-\frac{2 \mu \beta}{\hbar^{2}}-2 c m\right) x+\frac{2 \mu}{\hbar^{2}}\left(E_{0 \ell}-\gamma\right)+c^{2}=0
$$


if we equate corresponding coefficients of $x^{2}, x$ and $x^{0}$ respectively on both sides of Eq. (53), we have the following set of equations:

$$
\begin{aligned}
& m^{2}+b H m=\frac{2 \mu \alpha}{\hbar^{2}} \equiv \frac{\varepsilon_{1}^{2}}{r_{e}^{2}} \\
& b m-2 c m=\frac{2 \mu \beta}{\hbar^{2}} \equiv \frac{\varepsilon_{2}^{2}}{r_{e}^{2}} \\
& c^{2}=\frac{2 \mu}{\hbar^{2}}\left(\gamma-E_{0 \ell}\right)
\end{aligned}
$$

from Eq. (54) we find:

$$
m=-b H \lambda
$$

where the parameter $\lambda$ is given by:

$$
\lambda=\frac{1}{2}\left\{1+\left(1+\frac{4 \varepsilon_{1}^{2}}{b^{2} r_{e}^{2} H^{2}}\right)^{\frac{1}{2}}\right\}
$$

it is obvious that Eq. (58) leads to;

$$
\varepsilon_{1}=b r_{e} H\left(\lambda^{2}-\lambda\right)^{\frac{1}{2}}
$$

With $\mathrm{m}$ given by Eq. (57), Eq. (55) can be used to find the constant c, thus, our trial solution is well defined. The quantum correction can be evaluated using Eq. (10), which, when we use Eq. (26) to transform to variable $\mathrm{x}$ leads to:

$$
Q_{c}=-\frac{1}{b} \int_{x_{0 A}}^{x_{n B}} \frac{\varphi_{0 \ell}(x)}{\varphi_{0 \ell}^{\prime}(x)} k_{0 \ell}^{\prime}(x) \frac{d x}{x(1+H x)} .
$$

substituting Eq. (51) and Eq. (47) with $n=0$ in Eq. (60), get:

$$
Q_{c}=\frac{\varepsilon_{1}}{b r_{e}} \int_{x_{0 A}}^{x_{n B}} \frac{(x-c / m)(x+\beta / 2 \alpha)}{x(1+H x)} \frac{d x}{\sqrt{\left(x-x_{0 A}\right)\left(x_{0 B}-x\right)}}
$$

Eq. (61) can be expressed in partial fractions as:

$$
Q_{c}=\frac{\varepsilon_{1}}{b r_{e}} \int_{x_{0 A}}^{x_{n} B}\left\{\frac{1}{H}-\frac{\beta c}{2 \alpha m x}+\frac{(2 \alpha-\beta H)(m+c H)}{2 \alpha H m(1+H x)}\right\} \frac{d x}{\sqrt{\left(x-x_{0 A}\right)\left(x_{0 B}-x\right)}}
$$

the following standard integral (Dong and $\mathrm{Gu}, 2011)$ given by:

$$
\int_{x_{n A}}^{x_{n B}} \frac{d x}{(P+Q x) \sqrt{\left(x-x_{n A}\right)\left(x_{n B}-x\right)}}=\frac{\pi}{\sqrt{\left(P+Q x_{n B}\right)\left(P+Q x_{n A}\right)}}
$$

applied to Eq. (62) gives:

$$
Q_{c}=\frac{\pi \varepsilon_{1}}{b r_{e}}\left\{\frac{1}{H}-\frac{\beta c}{2 \alpha m} I_{1}-\frac{(2 \alpha-\beta H)(m+c H)}{2 \alpha H m} I_{2}\right\}
$$

where

$$
I_{1}^{-2}=x_{0 A} x_{0 B} \equiv \frac{\gamma-E_{0 \ell}}{\alpha} .
$$

Thus, by putting Eqs. (56) and (54) in Eq. (65), we find:

$$
I_{1}=\frac{\varepsilon_{1}}{c r_{e}}
$$

similarly; we find:

$$
I_{2}^{-2}=1+H\left(x_{0 A}+x_{0 B}\right)+H^{2} x_{0 A} x_{0 B}
$$

on substituting Eq. (42) and (43) in Eq. (67), this gives:

$$
I_{2}^{-2}=1-\frac{\beta H}{\alpha}+\frac{H^{2}\left(\gamma-E_{0 \ell}\right)}{\alpha} .
$$

by inserting Eqs. (54), (55) and (56) in (68) and simplifying, get: 


$$
I_{2}=\frac{\varepsilon_{1}}{r_{e}(m+c H)}
$$

substituting Eqs. (66) and (69) in (64) and employing (57) to eliminate $m$, we find:

$$
Q_{c}=\frac{\pi \varepsilon_{1}}{b r_{e}}\left(\frac{1}{H}+\frac{\varepsilon_{1}}{b r_{e} H^{2} \lambda}\right)
$$

The momentum integral on the left hand side of Eq. (7) is given in terms of variable $x$ as:

$$
I=-\frac{1}{b} \int_{x_{n A}}^{x_{n B}} k_{n \ell}(x) \frac{d x}{x(1+H x)}
$$

using Eq. (46) to eliminate $k_{n \ell}(x)$ in Eq. (71), we have:

$$
I=-\frac{\varepsilon_{1}}{b r_{e}} \int_{x_{n A}}^{x_{n B}} \frac{\sqrt{\left(x-x_{n A}\right)\left(x_{n B}-x\right)}}{x(1+H x)} d x
$$

In order to evaluate the integral in Eq. (72) we use the following standard integral obtained from Falaye et al. (2015)

$$
\int_{x_{n A}}^{x_{n B}} \frac{\sqrt{\left(x-x_{n A}\right)\left(x_{n B}-x\right)}}{x(1+Q x)} d y=\pi\left\{-\frac{1}{Q}-\sqrt{x_{n A} x_{n B}}+\frac{\sqrt{\left(Q x_{n A}+1\right)\left(Q x_{n B}+1\right)}}{Q}\right\}
$$

this give:

$$
I=\frac{\pi \varepsilon_{1}}{b r_{e}}\left\{\frac{1}{H}+\sqrt{x_{n A} x_{n B}}-\sqrt{\frac{\sigma}{H}+x_{n A} x_{n B}}\right\}
$$

where

$$
\sigma=\frac{1}{H}-\frac{\beta}{\alpha} \equiv \frac{1}{H}-\frac{\varepsilon_{2}^{2}}{\varepsilon_{1}^{2}}
$$

To find the rotational-vibrational energy eigenvalues of the effective improved Wei potential by EQR, we substitute Eq. (70) and (74) in Eq. (8) and replacing $N$ by $n+1$, this results to:

$$
\sqrt{y_{n A} y_{n B}}-\sqrt{\frac{\sigma}{H}+y_{n A} y_{n B}}=\frac{N b r_{e}}{\varepsilon_{1}}+\frac{\varepsilon_{1}}{b r_{e} H^{2} \lambda} \equiv \frac{b r_{e}(n+\lambda)}{\varepsilon_{1}}
$$

Thus, by solving Eq. (41) and (76), the rotational-vibrational energy is given by:

$$
E_{n \ell_{-} I W P}=\frac{\hbar^{2} \varepsilon_{3}^{2}}{2 \mu r_{e}^{2}}-\frac{\hbar^{2} b^{2}}{2 \mu}\left\{\frac{\sigma H \lambda(\lambda-1)}{2(n+\lambda)}-\frac{n+\lambda}{2}\right\}^{2}
$$

where $E_{n \ell \_}$IWP designates the rotational-vibrational energy of the improved Wei potential. Upon substituting Eqs. (29), (31) and (58) in (77) and replacing $H$ by $h^{\prime} e^{b r_{e}}$, this give:

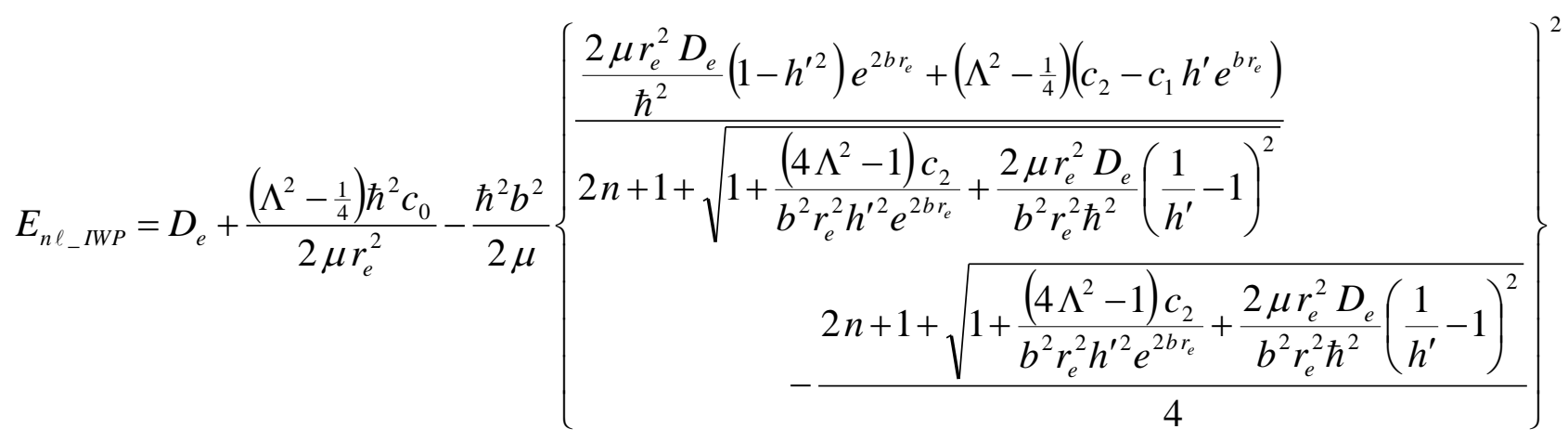

Evidently, Eq. (78) is only valid for $h^{\prime} \neq 0$, however, in the event that $h^{\prime}=0, E_{n \ell_{-} I W P} \rightarrow \infty$ which is not physically acceptable for a finite potential energy function. In order to deduce the rotational-vibrational energy eigenvalues of the 
improved Wei potential for the case $h^{\prime}=0$, first we observe that by letting $h^{\prime}=0$ in Eq. (14), the improved Wei potential reduces to:

$$
V_{M}(r)=D_{e}\left\{1-e^{-b_{M}\left(r-r_{e}\right)}\right\}^{2}
$$

which is the well-known Morse potential (Roy, 2013; Hamzavi et al., 2012), the subscript M in $V_{M}(r)$ and in bM designates "Morse". In subsequent notations, whenever the subscript M appears, it represents the Morse counterpart of the subject of discussion. Inputting $h^{\prime}=0$ in Eq. (16) gives:

$$
b_{M}=\pi c \omega_{e}\left(\frac{2 \mu}{D_{e}}\right)^{\frac{1}{2}}
$$

Next we will re-evaluate the quantum correction $Q_{c M}$ and the momentum integral $I_{M}$ and apply the EQR given by Eq. (8), with $h^{\prime}=0$ Eq. (61) gives:

$$
Q_{c M}=\frac{\varepsilon_{1}}{b r_{e}} \int_{x_{0 A}}^{x_{n B}}\left(x+\frac{\beta}{2 \alpha}-\frac{c_{M}}{m_{M}}-\frac{\beta c_{M}}{2 \alpha m_{M} x}\right) \frac{d x}{\sqrt{\left(x-x_{0 A}\right)\left(x_{0 B}-x\right)}}
$$

Eq. (81) can be evaluated by means of the following standard integrals (Ikhdair and Sever, 2009)

$$
\begin{aligned}
& \int_{x_{n A}}^{x_{n b}} \frac{x d x}{\sqrt{\left(x-x_{n A}\right)\left(x_{n B}-x\right)}}=\frac{1}{2} \pi\left(x_{n A}+x_{n B}\right) \\
& \int_{x_{n A}}^{x_{n b}} \frac{d x}{\sqrt{\left(x-x_{n A}\right)\left(x_{n B}-x\right)}}=\pi \\
& \int_{x_{n A}}^{x_{n b}} \frac{d x}{x \sqrt{\left(x-x_{n A}\right)\left(x_{n B}-x\right)}}=\frac{\pi}{\sqrt{x_{n A} x_{n B}}} \\
& \int_{x_{n A}}^{x_{n b}} \frac{\sqrt{\left(x-x_{n A}\right)\left(x_{n B}-x\right)}}{x} d x=\pi\left\{\frac{1}{2}\left(x_{n A}+x_{n B}\right)-\sqrt{x_{n A} x_{n B}}\right\}
\end{aligned}
$$

by applying the integrals given by Eqs. (82)- (84) on Eq. (81), we obtained:

$$
Q_{c M}=\frac{\pi \varepsilon_{1}}{b r_{e}}\left\{\frac{1}{2}\left(x_{0 A}+x_{0 B}\right)+\frac{\beta}{2 \alpha}-\frac{c_{M}}{m_{M}}-\frac{\beta c_{M}}{2 \alpha m_{M}} \frac{1}{\sqrt{x_{0 A} x_{0 B}}}\right\}
$$

using Eqs. (54), (55) and (56) with $h^{\prime}=0$ while $c$ and $m$ are replaced with $c_{M}$ and $m_{M}$ respectively, get:

$$
Q_{c M}=-\frac{\pi}{2}
$$

similarly, the momentum integral of Eq. (72) with $h^{\prime}=0$ gives:

$$
I_{M}=-\frac{\varepsilon_{1}}{b r_{e}} \int_{x_{n A}}^{x_{n B}} \frac{\sqrt{\left(x-x_{n A}\right)\left(x_{n B}-x\right)}}{x} d x
$$

the standard integral of Eq. (85) applied to Eq. (88) gives,

$$
I_{M}=-\frac{\pi \varepsilon_{1}}{b r_{e}}\left(\frac{\varepsilon_{2}^{2}}{2 \varepsilon_{1}^{2}}+\sqrt{x_{n A} x_{n B}}\right)
$$

with Eq. (89) and (87), the EQR of Eq. (8) gives:

$$
E_{n \ell_{-} M}=\frac{\hbar^{2} \varepsilon_{3}^{2}}{2 \mu r_{e}^{2}}-\frac{\hbar^{2} b^{2}}{2 \mu}\left(n+\frac{1}{2}+\frac{\varepsilon_{2}^{2}}{2 b r_{e} \varepsilon_{1}}\right)^{2}
$$

which upon substituting Eq. (29) and (30) in Eq. (90) gives the rotational-vibrational energies of Morse potential viz. 


$$
E_{n \ell_{-} M}=D_{e}+\frac{\left(\Lambda^{2}-\frac{1}{4}\right) \hbar^{2} c_{0}}{2 \mu r_{e}^{2}}-\frac{\hbar^{2} b^{2}}{2 \mu}\left\{n+\frac{1}{2}+\frac{\left(\Lambda^{2}-\frac{1}{4}\right) c_{1}-\frac{4 \mu r_{e}^{2} D_{e} e^{b r_{e}}}{\hbar^{2}}}{2 b r_{e} \sqrt{\left(\Lambda^{2}-\frac{1}{4}\right) c_{2}+\frac{2 \mu r_{e}^{2} D_{e} e^{2 b r_{e}}}{\hbar^{2}}}}\right\}^{2}
$$

\section{The radial eigenfunctions of the improved Wei potential}

To obtain the radial wave function corresponding to the rotational-vibrational energies of the improved Wei potential, we need to solve the Riccati equation given by Eq. (49), using the definition of the phase angle and the following transformation equation:

$$
z=1+H x
$$

Eq. (49) transforms to:

$$
z(1-z) \psi_{n \ell}^{\prime \prime}(z)+(1-2 z) \psi_{n \ell}^{\prime}(z)+\left(\varepsilon_{4}-\frac{\varepsilon_{5}}{z}-\frac{\varepsilon_{6}}{1-z}\right) \psi_{n \ell}(z)=0
$$

where

$$
\begin{aligned}
& \varepsilon_{4}=\frac{2 \mu \alpha}{b^{2} \hbar^{2} H^{2}} \\
& \varepsilon_{5}=\frac{2 \mu}{b^{2} \hbar^{2}}\left(\frac{\alpha}{H^{2}}-\frac{\beta}{H}+D_{e}-E_{n \ell}\right) \\
& \varepsilon_{6}=\frac{2 \mu}{b^{2} \hbar^{2}}\left(D_{e}-E_{n \ell}\right)
\end{aligned}
$$

in order to solve Eq. (96) we assume the following ansatz:

$$
\psi_{n \ell}(z)=N_{n \ell} z^{\tau}(1-z)_{2}^{v} F_{1}(-n, n+2 \tau+2 v+1 ; 2 \tau+1 ; z)
$$

where $N_{n \ell}$ is the normalization constant, $\tau$ and $v$ are constants and ${ }_{2} F_{1}$ is the hypergeometric function. Inserting Eq. (97) in (93), the resulting equation is Gaussian-hypergeometric if the following constraints are imposed:

$$
\begin{gathered}
\tau=\varepsilon_{5}^{\frac{1}{2}} \\
v=\varepsilon_{6}^{\frac{1}{2}}
\end{gathered}
$$

If $h^{\prime}=0$, the constants $\varepsilon_{5}$ and $\varepsilon_{6}$ becomes infinite, thus, making the wave function infinite which is not physically acceptable for a finite potential energy function. To obtain a physically acceptable wave function corresponding to $h^{\prime}=0$, Riccati Eq. (49) give for $h^{\prime}=0$ :

$$
x^{2} \psi_{n \ell}^{\prime \prime}(x)+x \psi_{n \ell}^{\prime}(x)+\left(-\varepsilon_{7} x^{2}+\varepsilon_{8} x-\varepsilon_{9}\right) \psi_{n \ell}(x)=0
$$

where

$$
\begin{aligned}
& \varepsilon_{7}=\frac{2 \mu \alpha}{b^{2} \hbar^{2}} \\
& \varepsilon_{8}=-\frac{2 \mu \beta}{b^{2} \hbar^{2}} \\
& \varepsilon_{9}=-\frac{2 \mu}{b^{2} \hbar^{2}}\left(D_{e}-E_{n \ell}\right)
\end{aligned}
$$

where we have used the definition of the phase angle $\varphi_{n \ell}(x)$ to recover the wave function $\psi_{n \ell}(x)$. Following Eyube $e t$ al. (2019a), Eq. (101) has solution of the form:

$$
\psi_{n \ell M}(x)=N_{n \ell M} e^{-\frac{1}{2} \xi x} x^{\frac{1}{2} \zeta}{ }_{1} F_{1}(-n, \zeta+1 ; \xi x)
$$

where the constants $\zeta$ and $\xi$ are respectively given by:

$$
\begin{aligned}
& \xi=2 \varepsilon_{7}^{\frac{1}{2}} \\
& \zeta=2 \varepsilon_{9}^{\frac{1}{2}}
\end{aligned}
$$




\section{RESULTS AND DISCUSSION}

Table 1 shows model parameters of three diatomic molecules studied in the present work, viz: $\mathrm{CO}, \mathrm{LiH}$ and $\mathrm{H}_{2}$. The data for $\alpha_{e}$ for all the three molecules were adopted from NIST data base. Data for $\mu, D_{e}, r_{e}$ and $\omega_{e}$ for CO were obtained from Yanar et al. (2020) and that of $\mathrm{LiH}$ and $\mathrm{H}_{2}$ were extracted from Ikhdair (2009)

Table 1: model parameters of diatomic molecules in the present study

\begin{tabular}{llll}
\hline parameter & \multicolumn{3}{c}{ molecule } \\
\cline { 2 - 4 } & $\mathrm{LiH}$ & $\mathrm{CO}$ & $\mathrm{H}_{2}$ \\
\hline$\mu(\mathrm{amu})$ & 0.8801221 & 6.85620871 & 0.50391 \\
$\mathrm{De}_{\mathrm{e}}\left(\mathrm{cm}^{-1}\right)$ & 20287 & 90670 & 38266 \\
$\mathrm{r}_{\mathrm{e}}(\AA)$ & 1.5956 & 1.12832320 & 0.7416 \\
$\omega_{\mathrm{e}}\left(\mathrm{cm}^{-1}\right)$ & 1405.5 & 2169.8129 & 4401.2 \\
$\alpha_{\mathrm{e}}\left(\mathrm{cm}^{-1}\right)$ & 0.2163911 & 0.01750513 & 3.0622 \\
\hline
\end{tabular}

Firstly, using Eqs. (16), (19) and (80), we have computed the values of the parameters $\mathrm{h}^{\prime}, \mathrm{b}$ and $\mathrm{b}_{\mathrm{M}}$ respectively for each of the diatomic molecule as indicated in Table 2. Also shown in Table 1 are the literature values of $b_{M}$ corresponding to $h^{\prime}=0$. For all the three diatomic molecules, our present result (PR) of $b_{M}$ is almost indistinguishable from those of Ikhdair (2009) and differ significantly from the values used by Roy (2013) owing to the fact that some of the molecular parameters $\left(\mu\right.$ and $\left.D_{e}\right)$ used by Roy (2013) are not in accord with Eq. (15). CO molecule has a relatively smaller $h^{\prime}(0.04)$ and $b \approx b$, therefore, the molecular properties of CO can approximately be described by Morse potential (see Figure 1), there is no need for a larger parameter potential such as the improved Wei or improved Tietz potential, $\mathrm{LiH}$ almost has the same trend as CO. However, for $\mathrm{H}_{2}$, the trend is different (see Figure 2)

Table 2: computed values of parameters $h^{\prime}, b$ and $b_{M}$ and corresponding literature values

\begin{tabular}{rllrrr}
\hline & \multicolumn{3}{c}{$\mathrm{h}^{\prime} \neq 0$} & \multicolumn{2}{c}{$\mathrm{bM}\left(\AA^{-1}\right), \mathrm{h}^{\prime}=0$} \\
\cline { 2 - 6 } molecule & $\mathrm{b}\left(\AA^{-1}\right)$ & $\mathrm{h}^{\prime}$ & $\mathrm{PR}$ & (Ikhdair, 2009) & $($ Roy, 2013) \\
\hline $\mathrm{CO}$ & 2.207914 & 0.040096 & 2.300141 & 2.2994 & 2.59441 \\
$\mathrm{LiH}$ & 1.066724 & 0.053791 & 1.127367 & 1.1280 & 1.7998368 \\
$\mathrm{H}_{2}$ & 1.722896 & 0.114178 & 1.944969 & 1.9426 & 1.440558 \\
\hline
\end{tabular}

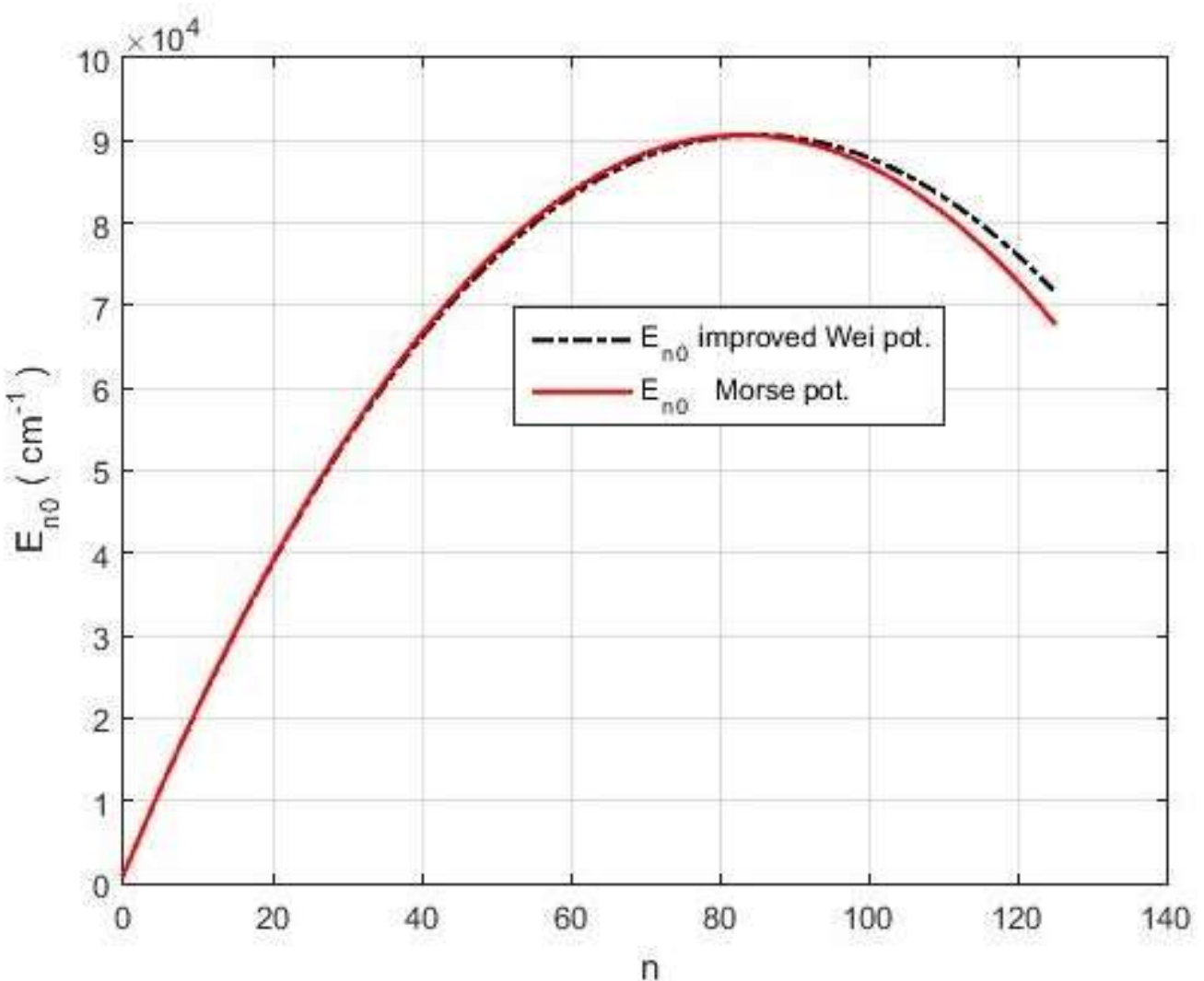

Figure 1 plot of pure vibrational energy of improved Wei potential for $\mathrm{h}^{\prime} \neq 0$ and for $\mathrm{h}^{\prime}=0$ as a function of $\mathrm{n}$ for CO molecule 


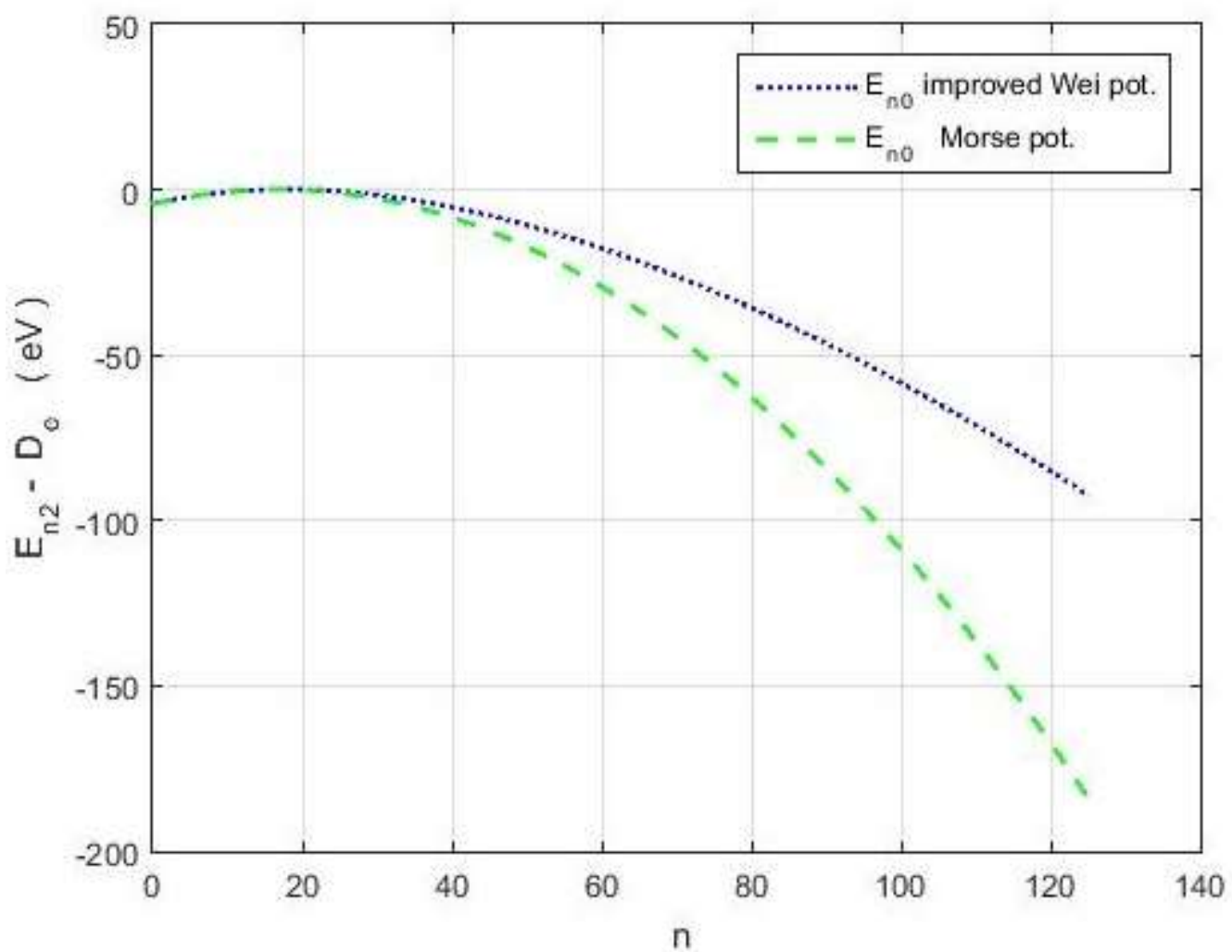

Figure 2 plot of d-state rotational-vibrational energy of improved Wei potential for $\mathrm{h}^{\prime} \neq 0$ and for $\mathrm{h}^{\prime}=0$ as a function of $\mathrm{n}$ for $\mathrm{H}_{2}$ molecule

In their work, Tang et al. (2014) have demonstrated how the improved Tietz potential fits the experimental Rydberg-KleinRees (RKR) data for two species of the Na2 diatomic molecules. Since the improved Wei potential is known to be an equivalent of the of the improved Tietz potential (Jia et al., 2012), it is quite logical to infer that the improved Wei potential can equally fits the RKR data, therefore, using Eqs. (78) and (91), we have computed pure vibrational state energies $(\ell=0)$ of the $\mathrm{CO}$ molecule for $\mathrm{h}^{\prime} \neq 0$ represented by $E_{n 0_{-} I W P}$ and for h' $=0$ represented by $E_{n 0_{-} M}$ respectively, to enable comparison, we have carried out our computation in three dimensions $(\mathrm{D}=3)$, the computed results are shown in Table 3. Also reported in the table are corresponding energies of the CO molecule adopted in the literature for the RKR data herein, represented by $E_{R K R}$ and that computed by Yanar et al. (2020) for the improved Tietz potential represented by $E_{n 0 \_} I T P$. To confirm the accuracy of our result we have calculated the mean absolute percentage deviation deviations ( $\sigma_{\text {mean }}$ ) defined as (Yanar et al., 2020; Tang et al., 2014):

$$
\sigma_{\text {mean }}=\frac{100}{N_{p}} \sum_{n=0}^{N}\left|\frac{E_{R K R}-E_{n 0}}{E_{R K R}}\right|
$$

where $N_{p}$ is the number of experimental data points. Eq. (107) applied to the data of Table 3 give: $0.2862 \%, 0.2896 \%$ and $0.0662 \%$ for improved Wei potential, improved Tietz potential and Morse potential respectively, the calculated mean absolute percentage deviations are each less than $1 \%$ as required by Lippincott criterion (Tang et al., 2014), these results show that the Morse potential can best fit the RKR CO data followed by improved Wei potential, then the improved Tietz potential, this is further evident from the result of our calculated value of $\mathrm{h}^{\prime}(\approx 0.04)$ which is relatively small, thus reducing the improved Wei potential to Morse potential. To further affirm the validity of our results, we have used Eqs. (78) and (91) to compute bound state energy of $\mathrm{H}_{2}$ and $\mathrm{LiH}$ for arbitrary values of $\mathrm{n}$ and $\ell$, to enable comparison with literature data, we have computed the differences $E_{n \ell}-D_{e}$ both for $\mathrm{h}^{\prime} \neq 0$ and $\mathrm{h}^{\prime}=0$, the results, along with corresponding literature results are presented in Table 4. For both $\mathrm{H}_{2}$ and $\mathrm{LiH}$, energies for improved Wei potential is in good agreement with those of Morse potential (obtained from Wei potential with $\mathrm{h}^{\prime}=0$ ) and with literature results, however, this agreement is more emphasized in $\mathrm{LiH}$ which has relatively smaller $h^{\prime}(0.054)$ than $\mathrm{H}_{2}$ with relatively larger $\mathrm{h}^{\prime}(0.114)$. 
Table 3: pure vibrational energies (in $\mathrm{cm}^{-1}$ ) of $\mathrm{CO}$ molecule of improved Wei (for $\mathrm{h}^{\prime} \neq 0$ and for $\mathrm{h}^{\prime}=0$ ) potential, improved Tietz potential and Rydberg-Klein-Rees data

\begin{tabular}{|c|c|c|c|c|}
\hline $\mathrm{n}$ & $E_{R K R}$ & $E_{n 0 \_I W P}$ & $E_{n 0 \_I T P}$ & $E_{n 0 \_M}$ \\
\hline 0 & 1081.7791 & 1081.6494 & 1081.6555 & 1081.6547 \\
\hline 1 & 3225.0522 & 3224.4172 & 3224.4230 & 3225.4922 \\
\hline 2 & 5341.8437 & 5340.1933 & 5340.1864 & 5343.3673 \\
\hline 3 & 7432.2200 & 7429.0182 & 7428.9867 & 7435.2799 \\
\hline 4 & 9496.2494 & 9490.9324 & 9490.8649 & 9501.2301 \\
\hline 5 & 11534.0013 & 11525.9762 & 11525.8618 & 11541.2178 \\
\hline 6 & 13545.5470 & 13534.1898 & 13534.0180 & 13555.2431 \\
\hline 7 & 15530.9592 & 15515.6133 & 15515.3743 & 15543.3059 \\
\hline 8 & 17490.3124 & 17470.2868 & 17469.9711 & 17505.4062 \\
\hline 9 & 19423.6825 & 19398.2502 & 19397.8488 & 19441.5441 \\
\hline 10 & 21331.1469 & 21299.5433 & 21299.0477 & 21351.7195 \\
\hline 11 & 23212.7846 & 23174.2059 & 23173.6081 & 23235.9325 \\
\hline 12 & 25068.6758 & 25022.2776 & 25021.5700 & 25094.1830 \\
\hline 13 & 26898.9019 & 26843.7980 & 26842.9736 & 26926.4711 \\
\hline 14 & 28703.5456 & 28638.8066 & 28637.8587 & 28732.7967 \\
\hline 15 & 30482.6901 & 30407.3427 & 30406.2651 & 30513.1598 \\
\hline 16 & 32236.4197 & 32149.4455 & 32148.2326 & 32267.5605 \\
\hline 17 & 33964.8189 & 33865.1543 & 33863.8009 & 33995.9987 \\
\hline 18 & 35667.9725 & 35554.5081 & 35553.0094 & 35698.4745 \\
\hline 19 & 37345.9652 & 37217.5459 & 37215.8976 & 37374.9878 \\
\hline 20 & 38998.8817 & 38854.3066 & 38852.5049 & 39025.5386 \\
\hline 21 & 40626.8057 & 40464.8291 & 40462.8704 & 40650.1270 \\
\hline 22 & 42229.8199 & 42049.1520 & 42047.0335 & 42248.7529 \\
\hline 23 & 43808.0060 & 43607.3139 & 43605.0331 & 43821.4164 \\
\hline 24 & 45361.4437 & 45139.3534 & 45136.9082 & 45368.1174 \\
\hline 25 & 46890.2106 & 46645.3088 & 46642.6976 & 46888.8560 \\
\hline 26 & 48394.3817 & 48125.2187 & 48122.4403 & 48383.6321 \\
\hline 27 & 49874.0291 & 49579.1211 & 49576.1747 & 49852.4458 \\
\hline 28 & 51329.2212 & 51007.0542 & 51003.9397 & 51295.2970 \\
\hline 29 & 52760.0226 & 52409.0562 & 52405.7736 & 52712.1857 \\
\hline 30 & 54166.4932 & 53785.1650 & 53781.7148 & 54103.1120 \\
\hline
\end{tabular}


Table 4: Bound state energy eigenvalues $(\mathrm{eV})$ for $\mathrm{H}_{2}$ and $\mathrm{LiH}$ of the improved Wei potential for $\mathbf{h}^{\prime} \neq \mathbf{0}$ represented by $E_{n \ell} I W P$ and for $\mathbf{h}^{\prime}=0$ represented by $E_{n \ell} M$ along with literature values, for arbitrary quantum states

\begin{tabular}{|c|c|c|c|c|c|c|c|c|c|}
\hline \multicolumn{2}{|c|}{ state } & \multicolumn{8}{|c|}{$\mathrm{LiH}$} \\
\hline $\mathrm{n}$ & $\ell$ & $E_{n \ell_{-} I W P}-D_{e}$ & $E_{n \ell_{-} M}-D_{e}$ & Roy (2013) & Ikhdair (2009) & $E_{n \ell_{-} I W P}-D_{e}$ & $E_{n \ell_{-} M}-D_{e}$ & Roy (2013) & Ikhdair (2009) \\
\hline & 0 & 4.47551366 & 4.47546399 & 4.47601313 & 4.47601 & 2.42889514 & 2.42889300 & 2.42886321 & 2.42886 \\
\hline & 1 & 4.41692266 & 4.42130175 & 4.46122852 & $\ldots$ & 2.42204173 & 2.42225366 & 2.42702210 & $\ldots$ \\
\hline & 2 & 4.30109884 & 4.31416214 & 4.43179975 & $\ldots$ & 2.40835920 & 2.40899771 & 2.42334244 & $\ldots$ \\
\hline & 5 & 3.64054831 & 3.70142462 & $\ldots$ & 4.25880 & 2.32693340 & 2.33008763 & $\ldots$ & 2.40133 \\
\hline 0 & 7 & 2.98090728 & 3.08685599 & $\ldots$ & $\ldots$ & 2.23997887 & 2.24577709 & $\ldots$ & $\ldots$ \\
\hline & 0 & 3.48838786 & 3.47824783 & 3.47991882 & $\ldots$ & 2.09941796 & 2.09848258 & 2.26054805 & $\ldots$ \\
\hline & 1 & 3.43433615 & 3.42757966 & 3.46633875 & $\ldots$ & 2.09283548 & 2.09208249 & 2.25875559 & $\ldots$ \\
\hline & 2 & 3.32742668 & 3.32732139 & 3.43932836 & $\ldots$ & 2.07969341 & 2.07930391 & 2.25517324 & $\ldots$ \\
\hline & 5 & 2.71618313 & 2.75311471 & $\ldots$ & $\ldots$ & 2.00147156 & 2.00322761 & $\ldots$ & $\ldots$ \\
\hline 2 & 7 & 2.10309849 & 2.17565220 & $\ldots$ & $\ldots$ & 1.91791494 & 1.92192947 & $\ldots$ & $\ldots$ \\
\hline & 0 & 3.04548267 & 3.02671113 & $\ldots$ & $\ldots$ & 1.94412680 & 1.94233203 & $\ldots$ & $\ldots$ \\
\hline & 1 & 2.99358880 & 2.97778999 & $\ldots$ & $\ldots$ & 1.93767791 & 1.93605155 & $\ldots$ & $\ldots$ \\
\hline & 2 & 2.89091795 & 2.88097239 & $\ldots$ & $\ldots$ & 1.92480234 & 1.92351165 & $\ldots$ & $\ldots$ \\
\hline & 5 & 2.30314419 & 2.32603114 & $\ldots$ & $\ldots$ & 1.84816046 & 1.84885224 & $\ldots$ & $\ldots$ \\
\hline 3 & 7 & 1.71226020 & 1.76712168 & $\ldots$ & $\ldots$ & 1.76627961 & 1.76906032 & $\ldots$ & $\ldots$ \\
\hline & 0 & 2.25750611 & 2.21778048 & $\ldots$ & 2.22052 & 1.65225713 & 1.64814021 & $\ldots$ & 1.64771 \\
\hline & 1 & 2.20972534 & 2.17235341 & $\ldots$ & $\ldots$ & 1.64607183 & 1.64209897 & $\ldots$ & $\ldots$ \\
\hline & 2 & 2.11513579 & 2.08241714 & $\ldots$ & $\ldots$ & 1.63372209 & 1.63003645 & $\ldots$ & $\ldots$ \\
\hline & 5 & 1.57215718 & 1.56600675 & $\ldots$ & 2.04355 & 1.56019781 & 1.55821082 & $\ldots$ & 1.62377 \\
\hline 5 & 7 & 1.02372251 & 1.04420339 & $\ldots$ & $\ldots$ & 1.48162401 & 1.48143131 & $\ldots$ & $\ldots$ \\
\hline & 0 & 1.59558520 & 1.53437350 & $\ldots$ & 1.53744 & 1.38509861 & 1.37809413 & $\ldots$ & 1.37756 \\
\hline & 1 & 1.55167317 & 1.49244051 & $\ldots$ & $\ldots$ & 1.37917229 & 1.37229212 & $\ldots$ & $\ldots$ \\
\hline & 2 & 1.46468732 & 1.40938557 & $\ldots$ & $\ldots$ & 1.36733920 & 1.36070697 & $\ldots$ & $\ldots$ \\
\hline & 5 & 0.96391735 & 0.93150602 & $\ldots$ & 1.37658 & 1.29687836 & 1.29171512 & $\ldots$ & 1.35505 \\
\hline 7 & 7 & 0.45557842 & 0.44680878 & $\ldots$ & $\ldots$ & 1.22155462 & 1.21794802 & $\ldots$ & $\ldots$ \\
\hline
\end{tabular}




\section{CONCLUSION}

In this research work, we have applied the ideas of exact quantization rule and ansatz solution method to obtain closed form expressions for the bound state rotational-vibrational eigensolutions of the $\mathrm{D}$-dimensional Schrödinger equation for the improved Wei potential, cases of $h^{\prime} \neq 0$ as well as $h^{\prime}=0$ were considered. We have computed rotational-vibrational energies for three diatomic molecules viz: $\mathrm{CO}, \mathrm{LiH}$ and $\mathrm{H}_{2}$ and compared our result with existing results in the literature. The results obtained in this work might be useful in areas of molecular physics, chemical physics, atomic physics and solid state physics.

\section{REFERENCES}

Dong, S-H. and Cruz-Irisson, M. (2012). Energy spectrum for a modified Rosen-Morse potential solved by proper quantization rule and its thermodynamic properties. $J$ Math Chem, 50; 881-892 doi.10.1007/s10910-9931-3

Eyube, E.S., Y.Y. Jabil and Umar, Wadata. (2019a). Bound State Solutions of Non-Relativistic Schrödinger Equation with Hellmann Potential within the Frameworks of Generalized Pekeris Approximation of the Centrifugal Term Potential. Journal of the Nigerian Association of Mathematical Physics 52; 215-222

Eyube, E.S., Sanda, A. and Y.Y. Jabil (2019b). $\ell$-wave analytical solutions of Schrödinger equation with Tietz-Hua potential. Journal of the Nigerian Association of Mathematical Physics 52; 223-230

Eyube, E.S., Yabwa, D. and Yerima, J.B. (2019c). Measurement of physical observables of a particle in a Morse potential. Transactions of the Nigerian Association of Mathematical Physics, 10; 51-60

Falaye, B.J., Ikhdair, S.M. and Hamzavi. M. (2015). Shifted Tietz-Wei oscillator for simulating the atomic interaction in diatomic molecules. Journal of Theoretical and Applied Mathematics, 9; 151-158

Falaye, B.J., Oyewumi, K.J., Ibrahim, T.T. Punyasena, M.A. and Onate, C.A. (2013). Bound state solution of ManningRosen Potential. Can. J. Phys., 91; 98-104 dx.doi.org/10.1139/cjp.2012-0330

Ferreira, F.J.S. and Bezerra, V.B. (2017). Some remarks concerning the centrifugal term approximation. Journal of Mathematical Physics, 58; 102104

Ferreira, F.J.S and Prudente, F.V. (2013). Pekeris approximation-another perspective. Physics Letters A, 377; 3027-3032

Greene, R.L. and Aldrich, C. (1976). Variational wave functions for a screened Coulomb potential. Physical Review A, 14; 2363-2366

Gu, X-Y. and Dong. S-H. (2011). Energy spectrum of the Manning-Rosen potential including centrifugal term solved by exact and proper quantization rules. J. Math Chem, 49; 2053-2062 doi./s10910-011-9877-5

Hamzavi, M., Rajabi, A.A. and Hassanabadi, H. (2014). The rotation-vibration of diatomic molecules with the Tietz-Hua rotating oscillator and approximation scheme to the centrifugal term. Molecular Physics. 110; 389-393 http://dx.doi.org/10.1080/00268976.2011.648962
Ikhdair, S.M. (2011). On the bound-state solutions of the Manning-Rosen potential including an improved approximation to the orbital centrifugal term. Physica Scripta, 83 (2011) $015010 \quad$ (10pp) doi.org/10.1088/0031$\underline{8949 / 83 / 01 / 015010}$

Ikhdair, S.M. (2009). Rotation and vibration of diatomic molecule in the spatially-dependent mass Schrödinger equation with generalized q-deformed Morse potential. $\begin{array}{lll}\text { Chemical } & \text { Physics, } & \text { 361; }\end{array}$ https://doi.org/10.1016/j.chemphys.2009.04.023

Ikhdair, S.M. and Sever, R. (2009). Exact quantization rule to the Kratzer-type potentials: an application to diatomic molecules. Journal of Materials Chemistry, 45; 1137 https://doi.org/10.1007/s10910-008-9438-8

Ikot, A.N., Awoga, O.A., Hassanabadi, H. and Maghoodi, E. (2014). Analytical approximate solutions of Schrödinger equation in D-dimensions with quadratic exponential-type potential for arbitrary $\ell$-state. Communications in Theoretical Physics, 61; 457-463

Jia, C-S., Diao, Y-F., Liu, X-J., Wang, P-Q., Liu, J-Y. and Zhang, G-D. (2012). Equivalence of the Wei potential model and Tietz potential model for diatomic molecules. The Journal of Chemical Physics. 137; 014101 (2012) http://dx.doi.org/10.1063/1.4731340

Khodja, A., Benamira, F. and Guechi, L. (2018). Path integral discussion of the improved Tietz potential. Journal of Mathematical Physics, 59; 042108 https://doi.org/10.1063/1.5022285

Khordad, R. and Mirhosseini. (2015). Application of Tietz potential to study optical properties of spherical quantum dots. Pramana Journal of Physics, 85; 723-737 http://dx.doi.org/10.1080/00268976.2011.648962

Kunc, J.A and Gordillo-Vazquez. (1997). Rotationalvibrational levels of diatomic molecules represented by the Tietz-Hua rotating oscillator. J. Phys. Chem. A 101; 15951602

Louis, H., Ita, B.I. and Nzeata, N.I. (2019). Approximate solution of the Schrödinger equation with Manning-Rosen plus Hellmann potential and its thermodynamic properties using the proper quantization rule. The European Physical Journal Plus, 134; 315 doi.org/10.1140/epip/i2019-12835-3

Lucha, W. and Schöberl, F.F. (1999). Solving Schrödinger equation for bound states with Mathematica 3.0 International Journal of Modern Physics, 10; 607-619. https://doi.org/10.1142/S0129183199000450

Ma, Z-Q. and Xu, B-W. (2005). Quantum correction in exact quantization rules. International Journal of Modern Physics E, 14; 599-610 doi.org/10.1142/s0218301305003429

NIST Computational Chemistry Comparison Benchmark Database NIST Standard Reference Database Number 101 Release 20, August 2019

Nasser, I., Abdelmonem, M.S. and Abdel-Hady, A. (2013). The Manning-Rosen Potential using J-matrix approach. Molecular Physics $\quad 3 ; \quad 1-8$ http://dx.doi.org/10.1080/00268976.2012.698026

Okorie, U.S., Ikot, A.N., Chukwuocha, E.O. and Rampho, G.J. (2020). Thermodynamic properties of improved 
deformed exponential-type potential for some diatomic molecules. Results in Physics, 17; 103978 http://doi.org/10.1016/j.rinp.2020.103078

Pekeris, C.L. (1934). The rotation-vibration coupling in diatomic molecules. Physical Review. 45; 98-103

Qiang, W-C., Chen, W-L., Li, K. and Wei, G-F. (2009). The scattering of the $\ell$-wave Schrödinger equation with second Pöschl-Teller-like potential. Physica Scripta, 79 (2009) 025005 (6pp) doi.org/10.1088/0031-8949/79/02/025005

Roy, A.K. (2013). Accurate ro-vibrational spectroscopy of diatomic molecules in a Morse oscillator potential. Results in Physics. 103-108 http://dx.doi.org/10.1016/j.rinp.2013.06.001

Serrano, F.A., Gu, X-Y. and Dong, S-H. (2010). Qiang-Dong proper quantization rule and its applications to exactly solvable quantum systems. Journal of Mathematical Physics, 51,082103

Tang, H.M., Liang, G-C., Zhang, L-H., Zhao, F. and Jia, C-S. (2014). Molecular energies of the improved Tietz potential energy. Can. J. Chem. 92; 201-205 dx.doi.org/10.1139/cjc2013-0466

Tsaur. G-Y. and Wang, J. (2014). A universal Laplacetransform approach to solving Schrodinger equation for all solvable models. Eur. J. Phys. 35; 015006 (17pp) doi.10.1088/0143-087/35/1/015006

Yanar, H., Tas, A., Salti, M. and Aydoddu, O. (2020). Rovibrational energies of $\mathrm{CO}$ molecule via improved generalized Pöschl-Teller potential and Pekeris-type approximation. The European Physical Journal plus 135; 292 http://dx.doi.org/10.1140/epip/s13360-020-00297-9

Yazarloo, B.H., Hassanabadi, H. and Zarrinkamar, S. (2012). Oscillator strength based on the Möbius square potential under Schrödinger equation. The European Physical Journal Plus, 127; 51 doi.org/10.1140/epjp/i2012-12051-9 use, distribution, and reproduction in any medium, provided the original work is cited appropriately. 